\title{
Annarosa Poli, Alla riscoperta di George Sand viaggiatrice in Italia
}

\section{Valentina Ponzetto}

\section{(2) OpenEdition}

10 Journals

\section{Édition électronique}

URL : http://journals.openedition.org/studifrancesi/529

DOI : 10.4000/studifrancesi.529

ISSN : 2421-5856

Éditeur

Rosenberg \& Sellier

\section{Édition imprimée}

Date de publication : 1 avril 2015

Pagination : 170-172

ISSN : 0039-2944

\section{Référence électronique}

Valentina Ponzetto, "Annarosa Poli, Alla riscoperta di George Sand viaggiatrice in Italia », Studi Francesi

[En ligne], 175 (LIX | I) | 2015, mis en ligne le 01 avril 2015, consulté le 18 septembre 2020. URL : http:// journals.openedition.org/studifrancesi/529; DOI : https://doi.org/10.4000/studifrancesi.529

Ce document a été généré automatiquement le 18 septembre 2020.

\section{(c) $(1) \&$}

Studi Francesi è distribuita con Licenza Creative Commons Attribuzione - Non commerciale - Non opere derivate 4.0 Internazionale. 


\title{
Annarosa Poli, Alla riscoperta di George Sand viaggiatrice in Italia
}

\author{
Valentina Ponzetto
}

\section{RÉFÉRENCE}

ANNAROSA POLI, Alla riscoperta di George Sand viaggiatrice in Italia, a cura di Emanuele KANCEFF, Moncalieri, C.I.R.V.I., 2010, «Biblioteca del viaggio in Italia», pp. 254.

1 Les études italiennes sur George Sand lui doivent tout, et les études sur les rapports multiples entre Sand et l'Italie sont indissolublement liées à son nom. Depuis son ouvrage magistral L'Italie dans la vie et dans l'œuvre de George Sand (Paris, Colin, 1960), dont la réédition en 2000 (Moncalieri, C.I.R.V.I., avec bibliographie mise à jour) a souligné, s'il en était besoin, l'indéfectible actualité, Annarosa Poli n'a cessé de consacrer une partie importante de son travail au réseau de liens qui unissent George Sand à notre pays, que ce soit par ses propres voyages, par le cadre et le personnel de ses œuvres, par ses rapports directs et épistolaires avec une foule d'intellectuels, d'hommes de lettres et de patriotes italiens.

À l'heure où l'Université de Vérone, où elle est professeur émérite, s'apprête à accueillir le colloque international organisé par Laura Colombo, George Sand et ses consœurs: la femme artiste et intellectuelle au XIX ${ }^{e}$ siècle (29 juin-1 ${ }^{\text {er }}$ juillet 2015), qui lui rendra hommage et sera placé sous son haut patronage, qu'il nous soit permis de signaler ce volume, sorti en 2010, qui a le mérite toujours d'actualité de ressembler et rendre disponibles quatorze articles publiés dans des revues italiennes et françaises sur une période de plus de cinquante ans, dont certains désormais difficiles d'accès.

3 Par une sorte d'hommage dans l'hommage et de mise en perspective de la place d'Annarosa Poli au sein de la communauté scientifique internationale, le recueil d'articles est précédé de plusieurs allocutions prononcées en Sorbonne le 5 avril 1995, lors d'une cérémonie en son honneur et de la présentation des mélanges George Sand et 
son temps, par Jean-Claude Fredouille, Michel Marat, Elio Mosele, Béatrice Didier, Pierre Brunel, Emanuele Kanceff et Annarosa Poli elle-même (pp. 8-40).

On regrettera que les articles soient disposés selon un ordre apparemment aléatoire que rien ne justifie, ni une notice éditoriale du responsable scientifique du volume, ni le sujet lui-même, ni l'ordre chronologique de composition. Il est d'ailleurs a fortiori dommage que rien, ni une note pour chaque article, ni une table liminaire, n'indique la date et le contexte de parution originale de chaque contribution, contraignant le sandien à avoir recours à d'autres sources pour se renseigner (pour la commodité du lecteur nous avons intégré ces renseignements à notre compte rendu). Ces péchés éditoriaux demeurent cependant véniels et n'altèrent en rien la valeur des articles, qu'on sera heureux de découvrir ou redécouvrir dans cette nouvelle présentation.

Ouvre le recueil un essai sur Il viaggio reale e il viaggio immaginario nelle "Lettres d'un voyageur" di George Sand (pp. 43-54, édition originale in La Letteratura e l'immaginario, 1984), qui remplit bien sa fonction liminaire, car il montre la superposition continuelle entre itinéraires et lieux réels et éléments d'imagination et de fictionnalisation littéraire dans les célèbres Lettres, démarche interprétative qu'on retrouvera dans d'autres articles du recueil. Dans une perspective bachelardienne, ce premier article analyse les symboles récurrents de la grotte, du bateau, de l'île et du rivage, et les mouvements de descente ou d'ascension comme autant d'éléments révélateurs $d u$ monde des rêves et de l'inconscient sandien.

Le modèle interprétatif de Bachelard, faisant appel au rêve comme porte de l'inconscient et à la symbolique des éléments, guide aussi deux autres essais du recueil, où dominent respectivement l'eau et le feu. Dans George Sand e la mitologia dell'acqua dolce: $i$ laghi italiani (pp. 125-140, in Goethe e Stendhal, mito e immagine del lago tra Settecento e Ottocento, 1988), l'eau, et en particulier l'eau douce des lacs et des rivières, apparaît tour à tour insidieuse et mortifère (comme dans Indiana ou La Mare au diable), ou source d'harmonie et d'apaisement, notamment dans le cas du Lac d'Iseo, cadre de l'action de Lucrezia Floriani et lié, selon le mythe, au culte d'Isis "dea madre, fecondatrice della terra e dell'acqua» (p. 131). "L'histoire du rêveur". Itinéraire fantastique à la recherche d'une écriture (pp. 203-216, in George Sand aujourd'hui, 1990), analyse ce roman inachevé de 1830 et son récit d'ascension à l'Etna sous le signe du feu et du voyage presque initiatique, mais surtout dans le processus d'apprentissage du métier d'écrivain par la romancière, pour arriver à saisir «le moment où [elle] ne se contente plus de raconter une histoire [...] mais cherche la manière la meilleure de la raconter» (p. 214).

7 Deux interventions, Lamennais à l'île de San Lazzaro de Venise: voyage réel ou voyage imaginaire? (pp. 55-66, in Viaggio immaginario, viaggio iniziatico, 1989) et Le père Aucher, flambeau européen de la culture arménienne, dans l'île de San Lazzaro de Venise (pp. 67-78, in À partir de Venise: héritages, passages, horizons, 2007), reviennent, grâce à des recherches dans les archives du couvent des Arméniens, sur la figure du père Pascal Aucher, le «frère Hiéronyme» des Lettres d'un voyageur. Le premier article démontre, à l'appui de lettres et autres documents, que la visite de Lamennais au couvent des Arméniens en 1832 fut bien réelle, ce qui ajouterait foi au fonds d'authenticité des échanges entre Sand et le moine à propos de l'abbé et de ses œuvres rapportés dans la III $^{\mathrm{e}}$ Lettre d'un voyageur. Le deuxième présente la figure du moine et ses rapports avec les plus célèbres voyageurs en visite sur l'île de San Lazzaro: Byron, lady Morgan, et encore George Sand et Lamennais. 
8 Un noyau consistant d'articles est lié à des aspects de ce qu'on peut appeler, selon la formule forgée par José-Luis Diaz, «le roman de Venise», c'est-à-dire les romanesques vicissitudes qui lièrent Sand, Musset et le docteur Pagello pendant le séjour à Venise de l'écrivaine en 1833-34. Ils sont tous caractérisés par une rigueur documentaire d'historien et presque de détective des lettres, et enrichis de quelques découvertes qui étaient inédites lors de la première publication des articles. Les Amants de Venise et Casanova (pp. 79-92, in «Revue d'histoire littéraire de la France», 1959), article important pour la recherche mussétienne, a le grand mérite d'avoir montré pour la première fois, par une argumentation à toute épreuve, que les annotations de Musset dites «notes de Venise» n'étaient pas des impressions de voyage, comme on l'avait longtemps cru, mais des notes de lecture prises sur les Mémoires de Casanova. Da Parigi a Genova. Album di memorie di Pietro Pagello con numerosi inediti (pp. 93-104, in «Letterature moderne», 1958) présente, à travers des citations extensives, dont l'intégralité de la Prefazioncella autographe de 1887, les souvenirs de la liaison Pagello-Sand que le médecin vénitien écrivit en 1835 mais ne laissa connaître que cinquante ans plus tard. La première réception, assez houleuse, de l'ouvrage est également évoquée. La Véritable histoire de la correspondance Sand-Pagello (pp. 105-114, in «Revue d'histoire littéraire de la France», 1957) retrace le tableau et l'histoire de la conservation et de la publication des très rares messages de Sand à Pagello qui nous sont parvenus, sept en tout en comptant les lettres proprement dites, la déclaration En Morée et le controversé billet au verso de la Canzonetta nuova. Plus loin dans le volume on trouve aussi l'intéressant et inattendu "Elle et Lui": realtà e letteratura (pp. 141-156, in Viaggiatori stranieri in Liguria, 1992), qui aborde le rapport entre autobiographie et fiction dans le roman à clé Elle et Lui dans la perspective exclusive et rafraîchissante de l'image de Gênes et de ses environs que Sand avait pu capter sur place lors de ses voyages de 1833 et 1855 et qu'elle retranscrit dans son œuvre.

Un autre noyau s'articule autour du voyage de 1855 , dont la destination principale est Rome, via Livorno, Pisa, Florence et Civitavecchia. Florence, ses artisans, pour lesquels Sand nourrit une grande admiration, et l'antiquaire Giovanni Freppa (1810-1868), qu'elle rencontra en 1855 et qui cherchait à retrouver le secret des maïoliques de la Renaissance, sont au centre de George Sand et les maïoliques florentines de Giovanni Freppa: la vertu de l'art populaire (pp. 115-124, in «Présence de George Sand», n. 27, octobre 1986). Tecnica e strutture narrative de "La Daniella" di George Sand (pp. 157-172, «Micromégas», anno IV, n. 1-2, 1977), au titre très référentiel, traite de la technique narrative à tiroirs, avec plusieurs histoires inclues l'une dans l'autre, et des séquences narratives qui constituent, selon l'A., autant de «stazioni mitiche dell'avventura dell'eroe» (p. 163) dans le roman La Daniella, dont A. Poli est une fine exégète puisqu'elle y a consacré deux éditions critiques et plusieurs articles. Plus strictement biographiques, deux autres articles traitent respectivement des lettres écrites par Sand lors de son séjour à Rome (Le Lettere di George Sand e il viaggio in Italia del 1855, pp. 173-180, in «Bollettino del C.I.R.V.I.», n. 2, 1980) et de la découverte de la campagne romaine, dont les souvenirs, consignés dans la correspondance et dans l'Agenda Mémento alimentent l'écriture de La Daniella (George Sand rifugiata nella campagna romana alla ricerca di un nuovo linguaggio, pp. 181-198, in L'Italia terra di rifugio, 2008). Suit la petite note George Sand e la memoria involontaria: ricordo del lago di Nemi (pp. 199-202, in «Bollettino del C.I.R.V.I.», n. 2, 1980), qui montre encore une fois l'intéraction féconde entre le réel, décevant et futile, et l'imaginaire, plus riche et spititualisé grâce au pouvoir de l'art. 
10 Le volume se referme sur une page à la mémoire d'Angiolo Tursi, le grand spécialiste du voyage en Italie à l'origine du fonds de la Biblioteca Marciana qui porte aujourd'hui son nom (Un caro e buon amico, pp. 217-218). Suivent les notes, regroupées en fin de volume en un bloc unique qui en rend la consultation un peu malaisée. Manque malheureusement une bibliographie des ouvrages cités dans le volume, qui aurait été souhaitable. 